\title{
Lettera ai lettori
}

Cari lettori, care lettrici,

con questo numero riprende la pubblicazione di Prisma, la rivista dell'Ires Marche.

Prisma ha rappresentato, per alcuni anni, uno strumento efficace e intelligente di diffusione di risultati di ricerca, riflessioni teoriche e pratiche operative sulle trasformazioni economiche e sociali delle Marche.

Intendiamo riprendere e rilanciare questo ambizioso progetto, con una nuova veste grafica, un nuovo editore e soprattutto con una nuova impostazione editoriale.

Oggi più che mai sentiamo il bisogno di avere quel luogo di incontro e dialogo tra ricerca, società civile, forze politiche e sociali, mondo dell'impresa, del lavoro e della cultura, che questa rivista ha rappresentato per la nostra regione. Oggi più che mai sentiamo il bisogno di promuovere una lettura critica delle trasformazioni economiche e sociali in atto, in particolare di quelle riguardanti il mondo del lavoro, attraverso il confronto con altre realtà a livello sia nazionale sia internazionale. Questa rivista si prefigge l'obiettivo "alto" di fornire chiavi di lettura che possano porsi all'attenzione della società e dell'economia locale; di favorire la crescita di una cittadinanza attiva e consapevole; di offrire, non da ultimo, uno strumento utile all'azione politica e sindacale, facilitando un confronto sulle ipotesi emergenti dai contributi critici e orientando prassi sempre più consapevoli.

La trasversalità delle competenze e il pluralismo delle chiavi di lettura costituiscono l'impianto metodologico di questo nuovo strumento di riflessione che, a partire da questo numero, mettiamo a disposizione della realtà regionale. Ma Prisma non sarà una rivista di tipo accademico, o per soli "addetti ai lavori”; tenterà, al contrario, di aprirsi a un pubblico più vasto di quello tradizionalmente raggiunto, proponendo articoli brevi e facilmente fruibili e sperimentando nuove forme espressive. In tale ottica abbiamo scelto di "incorniciare" i saggi di questa prima monografia tra due brevi "racconti". Le narrazioni di Ascanio Celestini e di Angelo Ferracuti, infatti, pungono e sollecitano la sensibilità del lettore sul tema della sicurezza del lavoro al pari delle attente disamine scientifiche ospitate in questo numero.

Il pluralismo e la multidisciplinarietà di Prisma trovano riscontro anche nella composizione del Comitato scientifico in cui, accanto a rappresentanti della ricerca umanistica e scientifica, figurano altrettanto autorevoli esponenti del mondo dell'economia, della cultura e dell'arte marchigiana.

Prisma è una rivista quadrimestrale, con due novità significative: il taglio monografico e la diffusione su scala nazionale, assicurata, quest'ultima, dall'esperienza editoriale 
della FrancoAngeli. In ogni numero, la sezione monografica verrà commentata da autorevoli discussant che, per il loro impegno in ambito politico, sindacale, imprenditoriale o amministrativo, possono offrire un contributo di esperienza al tema in discussione. Infine, la presenza di saggi "fuori dal tema", nonché di altre rubriche specifiche, è volta a promuovere un interesse che possa andare al di là della tematica monografica.

Dove trovare Prisma? La rivista, oltre che essere in vendita in libreria, è distribuita in abbonamento; abbiamo realizzato anche una versione on-line, che permette di accedere ai singoli articoli che più interessano.

A tutti voi, buona lettura.

Gianluca Busilacchi, Patrizia David, Gianni Venturi 